\title{
SFFS-SW: A Feature Selection Algorithm Exploring the Small-World Properties of GNs
}

\author{
Fábio Fernandes da Rocha Vicente ${ }^{1,2}$ and Fabrício Martins Lopes ${ }^{1}$ \\ 1 Federal University of Technology, Paraná, Brazil \\ 2 Institute of Mathematics and Statistics,University of São Paulo, São Paulo, Brazil \\ $\{$ fabiofernandes, fabricio\}@utfpr.edu.br
}

\begin{abstract}
In recent years, several methods for gene networks (GNs) inference from expression data have been developed. Also, models of data integration (as protein-protein and protein-DNA) are nowadays broadly used to face the problem of few amount of expression data. Moreover, it is well known that biological networks conserve some topological properties. The small-world topology is a common arrangement in nature found both in biological and non-biological phenomena. However, in general this information is not used by GNs inference methods. In this work we proposed a new GNs inference algorithm that combines topological features and expression data. The algorithm outperforms the approach that uses only expression data both in accuracy and measures of recovered network.
\end{abstract}

Keywords: small-world, gene networks, feature selection, graph theory, pattern recognition, bioinformatics.

\section{Introduction}

Complex networks systems is a very common phenomenon. In fact, we live in a universe of things that can be seen as complex networks [1 3]. These real systems are both biological and non-biological such as Internet, social interactions, physical systems, infection dynamics, regulatory networks, to cite but a few [3 7]. The occurrence of certain specific topologies has been observed and characterized in several research fields. These works points that diverse networks of natural phenomenon are not random but follow some particular arrangements [2-4, 8-10]. Thus, it is necessary to describe these distinct specific topologies in some manner to better understand the differences between them. In this way two aspects are important: characterization and representation [11]. A network can be characterized through a feature vector composed by network measurements, such as average vertex degree, average path length, degree distribution, etc., in which the network is said mapped to the feature vector. Thus, the feature vector can be used to group the several networks topologies into classes. On other hand, the inverse way is commonly impossible and the original network cannot be recovered from the feature vector. However, when the network can be recovered the mapping is said to provide a representation. Examples of representation are the adjacency list and the adjacency matrix [11, 12]. 
The study of complex networks can occur into two scenarios: In the first, the topology is determined by construction through a theoretical model thus, both the complete network and its properties can be clearly known a priori. The second group is composed by those networks for which the underlying construction rules (if one exists) are unknown and even the graph can be partially observed. In this case the topology is described in terms of its features. Frequently, both the rules of construction and the complete network are unknown as for instance, real neuronal network, gene networks (GNs) and social networks. The exception can occurs on planed networks as artificial neuronal networks, subway, power distribution, computer networks, etc.

When matter is life, the class of topology is commonly unknown. However, biological networks conserve properties between organisms and it is not very distinct of non-biological phenomenon [13]. Therefore, the research on this area try to determine some features that can characterize biological networks sometimes adopting some construction model as reference. Distinct descriptions has been proposed to biological networks as scale-free [2] for example in yeast [13] and metabolic networks [13]. Also a hierarchic structure was observed in E.coli [14]. In special, the small-world (SW) topology [15, 16] has been observed in several areas and seems to be a common phenomenon both in physical events, social and biological networks. Some examples are: seismic events 7], subway station distribution 5], social networks [15, 17], value dynamics in financial market [18], epidemics [19], neuronal networks [4] and brain networks [6].

In particular, it was adopted in this work the SW construction model proposed by Watts and Strogatz [20, 16]. This model has two main properties: small average path length and high average clustering coefficient. These SW features has been observed on biological networks. For instance human protein-protein interaction network (PPI) presented SW properties [21] and a study of networks of 43 organisms presented a higher clustering coefficient [22].

The research of gene networks is broadly used to better understand living organisms. Moreover, since many gene interactions remains unknown, the inference of gene networks from expression data has been used to discover new interactions [23 27]. Moreover, the topology of living organisms should conserve some structure, following some particular features. Considering that the inference of gene networks is an inverse problem where more than one network could produce equivalent data [28, 26], the use of topology could help the search algorithms by avoiding improbable biological structures. An algorithm guided by scale-free (Barabási-Albert [2]) topology achieved better accuracy on inference [29]. Thus, the topology can be an important component for GNs inference. This work presents a new algorithm for GNs inference whose criterion function is based both in expression data and topological features of SW networks.

\section{Methodology}

The real structure of gene networks (GNs) is commonly unknown and the observation of physical relationships between components (protein-DNA, proteinprotein, etc.) is expensive and some times hard to obtain. Therefore, the network 
is frequently inferred from expression. The reasoning behind this approaches is that the set of relationships between cellular components (i.e. the network) produces the output observed expression. The challenge is to recover the network from the observed data [23, 26]. It was adopted in this work the Probabilistic Boolean Networks (PBN) model [23] to represent the gene network. PBN is a probabilistic approach derived from Boolean Networks (BN) model, first introduced by Kauffman [30]. The genes of a BN are represented by variables and can just assume discrete values, typically 1 (the gene is up regulated) and 0 (the gene is down regulated). In other words, the value correspond to the expression of that gene in a given observation $t$. The value of one given variable in the sample $t$ is named state of the variable and the set of values of all variables in the sample $t$ is called state of the system. The relationship between genes is represented by boolean functions. If the samples is given through a time series, the set of boolean functions applied to the system state at observation $t$ determines the state of the system in the next observation $t+1$. Thus, the transitions between states can be deterministic $(\mathrm{BN})$ if there is no changes in the set of boolean functions or probabilistic $(\mathrm{PBN})$ where each variable is associated to set of boolean functions with a probability of choice at each observation.

In this work we address the problem of selecting a set of predictor genes (i.e. features) in the sample $t$ that can better be used to classify the state value of a given target gene on a sample $t+1$ based on the state of the set of predictors in the time sample $t$. In this context, a naive algorithm could perform an evaluation over all possible combinations of $n-1$ genes taken $k$ at time, with $k=1,2, \ldots, n-1$, leading to an exponential increasing of the search space with the increment of $n$. Ir order to avoid this computational complexity we have adopted a feature selection method.

\section{Feature Selection Algorithm}

Regarding the classification task, a feature selection approach try to select a subset of features that produce the better classification of the observed classes. In this way, a feature selection algorithm requires two components: a criterion function that assign a value to a subset of features and a search algorithm whose objective is performed in order to find a subset of features that minimize/maximize the criterion function. The SFFS algorithm [31] and other alternative versions to the canonical SFFS has been applied on GNs inference [32, 33]. Thus, we adopted the SFFS search strategy on this work.

\section{Criterion Function}

We defined a criterion function that includes both the gene expression data and a topological features. The expression values are used to compute the Mean Conditional Entropy (MCE) which is presented below. 
Mean Conditional Entropy. In some problems it is necessary to assign a measure of uncertainty to a given random variable $Y$ after the observation of another random variable $X$. For instance, the uncertainty about the value of a target gene $Y$ after the observation of the value of another gene $X$. The conditional entropy of $Y$ given $x$ is defined as:

$$
H(Y \mid x)=-\sum_{y \in Y} P(y \mid x) \log [P(y \mid x)]
$$

The Mean Conditional Entropy [32] is the weighted average of $H(Y \mid x)$ for each $x \in X$.

$$
H(Y \mid X)=\sum_{x \in X} H(Y \mid x) P(x)
$$

In the common context of GNs inference, the algorithm must search for those variables (i.e. features) that minimizes the $\mathrm{MCE}$ even if using few observations. Furthermore, even one had large observation set, it could be expected that some system states does not occur because they can be rare [26, 34, 23]. Thus, the number of observed instances $(N)$ is commonly much lower then the number of possible instances $(M)$. Thereby, to face this problem the MCE is computed with penalization of the non-observed instances as defined in [32]:

$$
H(Y \mid X)=\frac{\alpha(M-N) H(Y)+\sum_{i=1}^{N}\left(f_{i}+\alpha\right) H\left(Y \mid X=x_{i}\right)}{\alpha M+T}
$$

where $T$ is the number of samples, $f_{i}$ the absolute frequency of $x_{i}$ and $\alpha$ is a parameter to determine the weight of the penalization.

Small-World Networks. The small-world network proposed by Watts and Strogatz [20] allows to model the increasing of randomness in a regular network. The SW construction proposed by Watts and Strogatz is defined as follows: First set $N$ as the number of vertices and $k$ as the average degree. Start with a one-dimensional ring lattice with each vertex connected to $2 k$ neighbors. Then, for each vertex rewire each edge with probability $p$. Thus, $p$ defines the global randomness of the network which ranges from $p=0$ (regular original lattice) to $p=1$ (totally random).

The two main features of this networks are the clustering coefficient $(C)$ and path length $(L)$. The clustering coefficient (Eq. 4) of a vertex $C_{v}$ is defined as follows: given a vertex $v$, select its $m$ neighbors. Then, count the number of edges $\eta_{v}$ between the $m$ neighbors of $v$ (i.e. except the edges with $v$ ). Let $\eta_{m}=m(m-1) / 2$ denote the maximum number of edges in the sub-graph with $m$ vertices. Then, compute $C_{v}=\eta_{v} / \eta_{m}$. Thus, the network clustering coefficient is given by averaging over all vertices:

$$
C=\frac{1}{N} \sum_{v \in V} C_{v}
$$


The path length $L_{i j}$ is defined as the number edges in the shortest path between two vertices $v_{i}$ and $v_{j}$. The network path length $L$ is the average over all pair of vertices. Let $\lambda$ denote the number of paths in the network.

$$
L=\frac{1}{\lambda} \sum_{v_{i}, v_{j} \in V} L_{i j}
$$

MCE-SW Criterion Function. We defined a criterion function based on the MCE and the topological properties of SW networks (MCE-SW). The MCESW is a linear combination of MCE and SW features. The topological part is composed by the two SW features described by Watts and Strogatz [16].

The clustering coefficient is in the interval [0,1] as defined on Equation 4. The path length $L$ is a value greater than 1 without upper limit since $N$ is a network parameter. The theoretical maximum path length for a given pair $v_{i}, v_{j}$ is $N-1$ in a network of size $N$. However, the path length in a SW network is frequently very lower than that maximum. Thus, $L$ is normalized through max-min normalization (Eq. 6). Where $\min =1$ and $\max$ was estimated by sampling $1000 \mathrm{SW}$ networks with the same $k, N$ and $p$ and taking the maximum path length. Also, if eventually $L$ is greater than $\max$ than we set $L=1$.

$$
\operatorname{Normalized}(L)=\frac{L-\min }{\max -\min }
$$

Form this point we will refer to the Normalized $(L)$ simply as $L$. The MCESW criterion function has one parameter $w \in[0,1]$ which is the weight of topological features. We set half of $w$ to each topological measure $w_{1}=w / 2$ and set weight of MCE $w_{2}=1-w$. Thus, the MCE-SW criterion function of two variables $x$ and $y$ is defined as:

$$
\begin{aligned}
\mathrm{MCE}-S W_{y, x} & =w_{2} \times M C E_{x, y}+w_{1} \times L-w_{1} \times C \\
& =w_{2} \times M C E_{x, y}+w_{1} \times(L-C)
\end{aligned}
$$

Where $C \in[0,1]$ and $L \in[0,1]$. Since, $L-C \in[-1,1]$ we rescale $(L-C)$ in $[0,1]$ to maintain a positive score.

$$
S W=\frac{(L-C)+1}{2}
$$

Thus, MCE-SW became:

$$
\mathrm{MCE}-\mathrm{SW}_{y, x}=w_{2} \times M C E_{x, y}+w_{1} \times S W
$$

\section{SFFS-SW Feature Selection Algorithm}

The proposed search algorithm is composed by two distinct steps. At the fist step the network is inferred from expression data by using MCE as criterion 
function, which guarantees an initial topology. On the second step each vertex is revisited at the same order they were visited in the first phase and the MCESW criterion function is applied. As can be seen in Equation 9 the part MCE gives the local contribution of the edges between a gene $y$ and its predictors. In other words, MCE does not take into account how the the global topology if affected by those edges. Differently, the topological part measures how the local changes influences the global properties of the network. Thus, the second phase of the algorithm readdress the inferred edges in terms of both local and global topological effects.

The Algorithm 1 receive $N$ genes $G$, the expression data set $D$, the maximum number of features $m f$ the SFFS will search for each target $Y$ and the weight of topological measurements $w$. Once the set or predictors of a target gene $Y$ is defined by SFFS-SW algorithm, the edges from predictors to target are added and thus, the network is updated. At each iteration of the second step

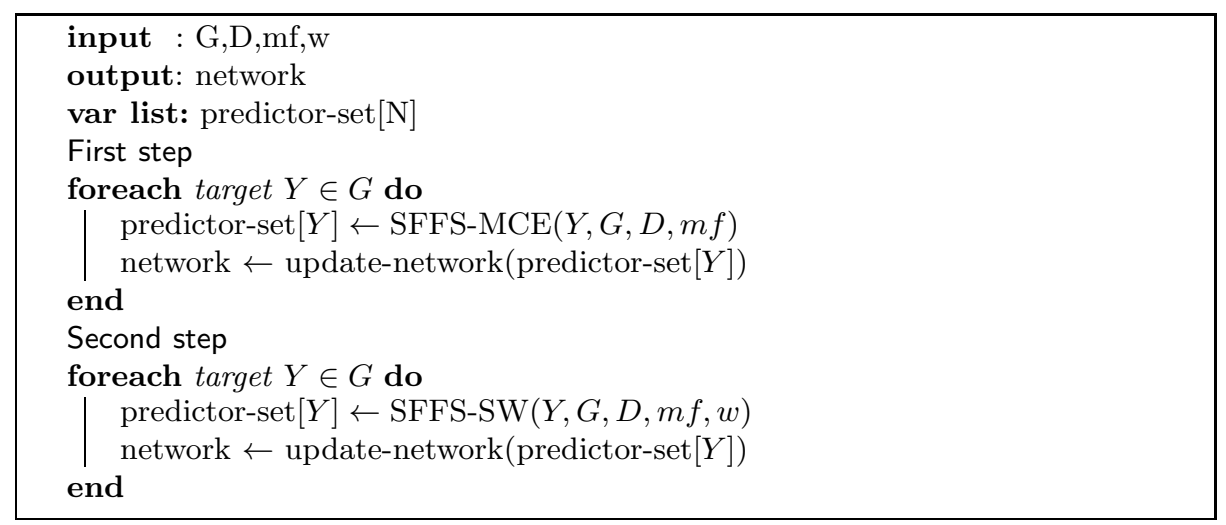

Algorithm 1. Main function of the inference algorithm

of Algorithm 1, the SFFS method is performed to search for the best subset of size $q \leq m f$. The edges from a feature set $X$ to the target gene $Y$ are temporarily added to the graph in order to compute the topological features and the criterion function. After the criterion function is computed the edges are removed. At the end, when the best subset is finally chosen the edges are permanently added in the network. The Algorithm 2 shows the computation of the criterion function value for a given feature set $X$. The algorithm starts by removing all previously inferred predictor of that target variable in the first step of Algorithm 11 The inclusion of each feature on this execution take into account how much the added characteristic affects both the MCE and the network topology by considering all other previously inferred edges. In other words, the predictor set of each target is re-inferred from an empty set, based on the possible changes in topology. 


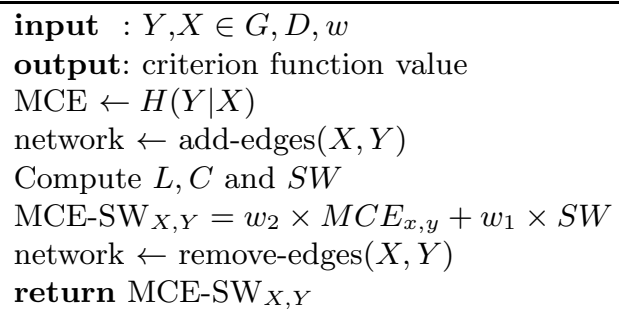

Algorithm 2. MCE-SW criterion function computation

Computational Complexity. Assuming a network with $N$ vertex and maximum degree $k$, the complexity of SFFS algorithm is performed $N$ times (one for each target), each with cost $O\left(2^{k}\right)$. The complexity of computation of the average clustering coefficient part depends on the number of neighbors $k_{v}$ of each vertex $v$. The algorithm must to sum the number of edges between the $k_{v}$ neighbors of $v$. Thus, the maximum number of operation for a given vertex is $k^{2}$, The shortest path between two vertices is computed in $O\left(N^{2}\right)$ and the average shortest path is computed to $N$ vertex in $O\left(N^{3}\right)$. Thus, for each subset of predictors (or for each target) the algorithm calculates the average clustering coefficient and average path length procedures performing $k^{2}+N^{3}$ operations. Thus, the SFFS performs $2^{k} \times\left(k^{2}+N^{3}\right)$ operations. Since in the context of GNs inference $k$ is limited to a small value the total cost of one run of SFFS algorithm is $O\left(N^{3}\right)$. The SFFS algorithm is executed $N$ times (one for each target) on each step of algorithm. Thus the total complexity of algorithm is $O\left(N^{4}\right)$. The computational cost is justified by the increasing in the performance on recovering a network with better topological features.

Validation. In order to evaluate the method we used the Artificial Gene Networks (AGN) simulation and validation model 35, 36] to generate both the networks and the expression data. The AGN was used to create Watts and Strogatz SW networks and to simulate the corresponding output signal by considering a probabilistic boolean network model. To evaluate the performance of the proposed algorithm we computed the following confusion matrix:

Table 1. Confusion matrix. $\mathrm{TP}=$ true positive, $\mathrm{FN}=$ false negative, $\mathrm{FP}=$ false positive, $\mathrm{TN}=$ true negative.

\begin{tabular}{c|c|c} 
Edge & Inferred & Not Inferred \\
\hline Present & TP & FN \\
Absent & FP & TN
\end{tabular}

Commonly, in GNs inference it is preferable to predict a low number of edges with high precision than a high number of edges with low precision. This has a practical aspect: the inferred relationships will probably be validated through 
some expensive biochemical experiment. Thus, the set of predicted edges can be as a set of hypothesis to be tested. However, an ideal algorithm model should recover edges with high precision without missing existing edges. To evaluate these properties we compute the PPV (Positive Predictive Value, also know as precision) and Sensitivity (also know as recall or True Positive Rate - TPR). We adopted also the similarity measure which is a geometrical average of precision and recall.

$$
\begin{aligned}
P P V & =\frac{T P}{(T P+F P)} \\
\text { Sensitivity } & =T P R=\frac{T P}{(T P+F N)} \\
\text { Similarity }(A, B) & =\sqrt{P P V \cdot \text { Sensitivity }}
\end{aligned}
$$

Since we are mostly interested on the inference of networks that follow smallworld properties, we also adopted the computation of trajectories for both clustering coefficient and path length at each step of the inference algorithm.

\section{Results and Discussion}

This section presents the experimental results of the proposed methodology when applied to SW networks. We evaluate our methods in networks with 100 vertices and average edges $k$ varying from 1 up to 4 . We set the rewiring probability to $p=0.01$ and the threshold of the criterion function to 0.3 . We performed 10 executions to each configuration. On each execution produced a network in the first phase (recovered-1). Then, the second step of the proposed algorithm was executed with weights $0.2,0.4,0.6,0.8,1.0$. For each weight the search always starts from the same recovered-1 network of that configuration.

It is important that the topological features do not suppress the expression information but on the contrary, be well combined in order to increase the accuracy and the networks properties. We find a small variation in accuracy as the topological information weight vary from $0.2-0.6$ (Figure 1).

The algorithm frequently achieves the highest precision values when $w=0.8$ (see Table 2). There is a gain in precision given by the inclusion of topological information for any value of $k$. It is interesting to note that when the weight is 1.0 the PPV decreases fast showing that both expression and topological information are essential to inference. There is also an improvement in sensitivity when SW features is used. The exception occurs in the highest connected network $(\mathrm{k}=4)$ were the value is not altered. Thus, this also suggests an interdependence between topology and the expression. Moreover, the topological features does not suppress the expression information, but in contrary, the search is biased through the combination of these two elements. the inference is not dominated by topological features it is just biased by the new criterion function.

We also analyzed the trajectory of clustering coefficient $(C)$ and path length $(L)$ - Figure 2. It could be observed distinct behaviors between $C$ and $L$ and also an improvement on the small-world features of the recovered network. 


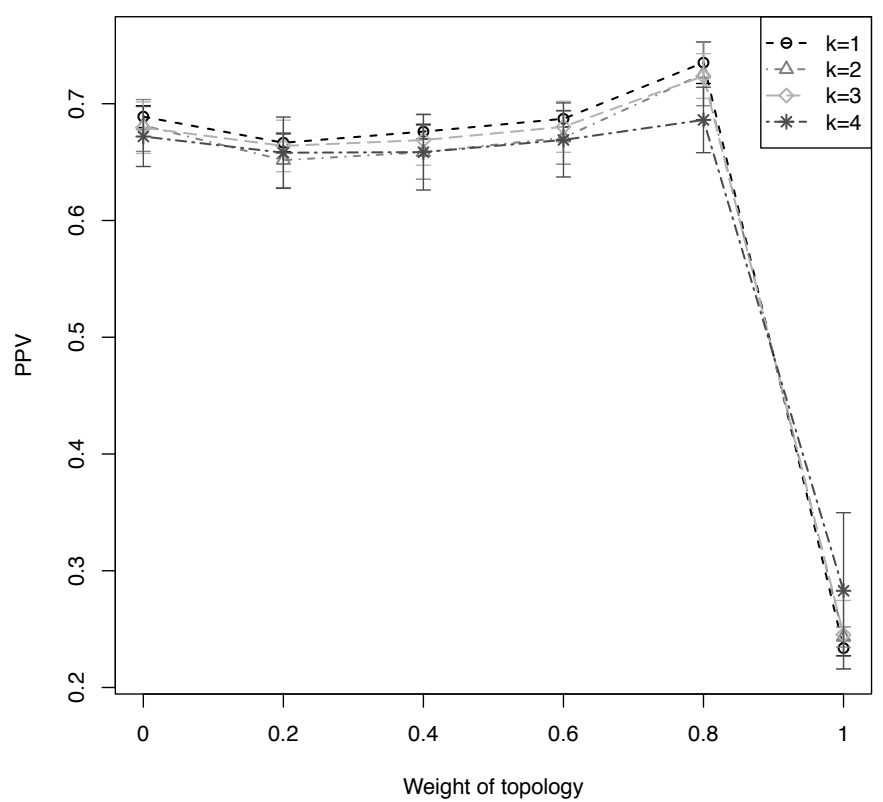

Fig. 1. Positive Predictive Value (PPV). The algorithm reaches the highest value with $\mathrm{w}=0.8$ for any $\mathrm{K}$. The precision decreases fast when the algorithm does not consider the MCE $(\mathrm{w}=1.0)$ showing that both expression and topology is necessary to increase precision.

Table 2. Precision (PPV), Sensitivity (Recall) and Similarity for $k=1,2,3,4$ and weight $=0,0.2,0.4,0.6,0.8,1.0$

\begin{tabular}{|c|c|c|c|c|c|c|c|}
\hline & \multicolumn{6}{|c|}{ weight } \\
\hline & & 0 & 0.2 & 0.4 & 0.6 & 0.8 & \\
\hline \multirow{3}{*}{$\mathrm{k}=1$} & $\overline{\mathrm{PPV}}$ & 0.56 & 0.54 & 0.55 & 0.56 & 0.60 & 0.10 \\
\hline & Sensitivity & 0.78 & 0.80 & 0.80 & 0.80 & 0.80 & \\
\hline & Similar & $\overline{0.76}$ & 0.75 & $\overline{0.76}$ & 0.76 & 0.78 & 0.20 \\
\hline \multirow{3}{*}{$\mathrm{k}=2$} & $\mathrm{PPV}$ & 0.60 & 0.57 & 0.58 & 0.59 & 0.65 & 0.1 \\
\hline & Sensit & 0.58 & 0.59 & 0.58 & 0.58 & 0.58 & 0.0 \\
\hline & Simil & 0.70 & 0.69 & 0.69 & 0.70 & 0.72 & 0.18 \\
\hline \multirow{3}{*}{$\mathrm{k}=3$} & PPV & 0.62 & 0.60 & 0.61 & 0.62 & 0.67 & 0.13 \\
\hline & Sensit & 0.44 & 0.45 & 0.45 & 0.44 & 0.44 & 0.10 \\
\hline & Similar & 0.65 & 0.64 & 0.64 & 0.65 & 0.66 & 0.10 \\
\hline \multirow{3}{*}{$\mathrm{k}=4$} & $\overline{P P V}$ & 0.67 & 0.66 & 0.66 & 0.67 & 0.69 & 0.28 \\
\hline & Sensit & 0.39 & 0.39 & 0.39 & 0.39 & 0.35 & 0.01 \\
\hline & Simil & 0.64 & 0.64 & 0.63 & 0.64 & 0.62 & 0.12 \\
\hline
\end{tabular}



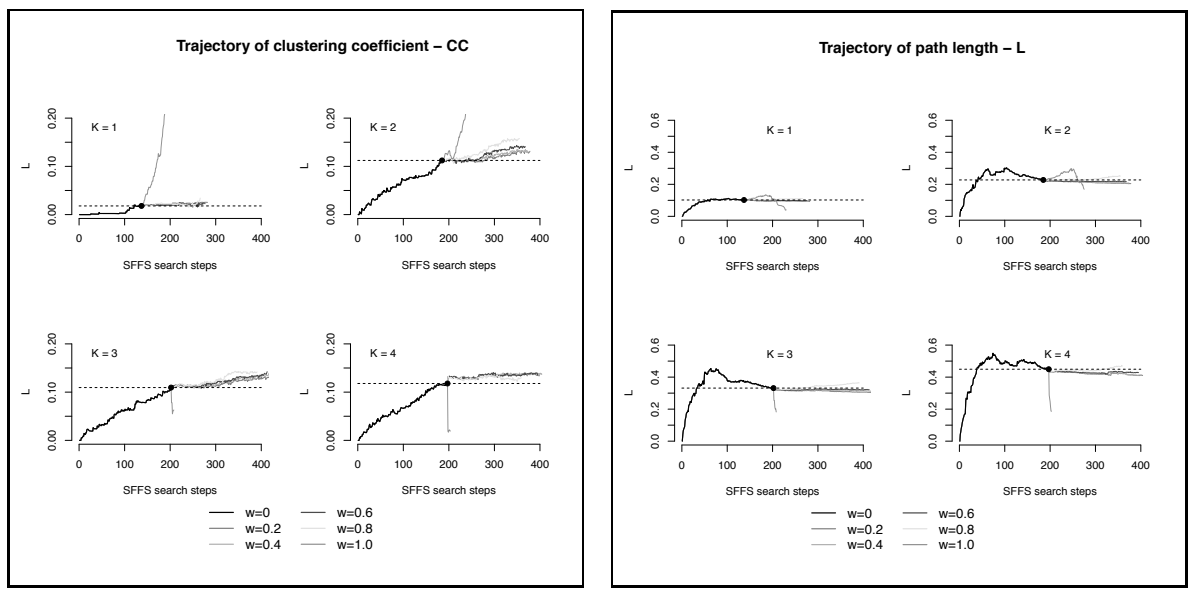

Fig. 2. Left: trajectory of the average clustering coefficient. The first step of the algorithm (SFFS-MCE) is printed in black. The achieved value of the SFFS-SW step is higher than the final value of the SFFS-MCE step (exceptions: weight $=1.0$ with $k=3$ and $k=4$ ). Right: trajectory of the average path length (normalized in the range $[0,1])$. The SFFS-SW achieves a lower value than the SFFS-MCE. The exception is weight $=0.8$.

With respect to clustering coefficient $C$ the value starts from zero, gradually increases until it achieves a point. In the second step the value increases when SFFS-SW is applied, showing the relevance of this topological feature.

With respect to path length, the algorithm presented a distinct behavior. Like $C$, on the first phase $L$ starts from zero. However, it can be observed that $L$ increases fast at the first steps then, it decreases and converges to an smallest path length. This shows that the SFFS-MCE algorithm drives the search to a small path length. Following this reasoning, the bias given by the new criterion function drives the path length to a lower value. However, the trajectory maintains almost stable in respect to path length.

The MCE-SW criterion function improve the features of the inferred network by increasing the clustering coefficient and maintaining the achieved path length. The algorithm reach different values as average degree increases.

\section{Conclusion}

In this work we presented a new feature selection method, called SFFS-SW for the inference of GNs. The algorithm uses both expression data and two topological features of SW networks: high average clustering coefficient and low average path length. The SFFS-SW combines the Mean Conditional Entropy (MCE), network clustering coefficient and the network path length through a criterion function. The search is performed through an SFFS algorithm in two steps: The first step uses only expression to recover a network. On the second step 
the network is inferred by considering both MCE and SW features. The proposed algorithm inferred networks with higher precision and recall than the strategy that uses only expression data. Moreover, the inferred networks are biased to topology with higher clustering coefficient and lower average path length than networks inferred without topological information. Thus, even when precision and recall are very similar between topological and non-topological approaches, the network features are biased to SW topology in SFFS-SW. The reasoning is that it is important not only to find a set of correct edges and discard incorrect ones, but to find those correct edges that are also consistent to the network topology. Finally, the results indicate that topological information is important both on the inference process and evaluation of results.

Acknowledgement. We would like to thanks the financial support of FAPESP grant 2011/50761-2, CNPq, CAPES, NAP eScience - PRP - USP, and Fundação Araucária.

\section{References}

1. Latora, V., Marchiori, M.: Efficient Behavior of Small-World Networks. Physical Review Letters 87(19), 198701 (2001)

2. Albert, R., Barabási, A.L.: Statistical mechanics of complex networks. Reviews of Modern Physics 74(1), 47-97 (2002)

3. Easley, D., Kleinberg, J.: Networks, Crowds, and Markets: Reasoning about a Highly Connected World. Cambridge University Press (2012)

4. Lago-Fernández, et al.: Fast response and temporal coherent oscillations in smallworld networks. Physical Review Letters 84(12), 2758 - 2761 (March 2000)

5. Latora, V., Marchiori, M.: Is the Boston subway a small-world network? Physica A: Statistical Mechanics and its Applications 314(1-4), 109-113 (2002)

6. Bassett, D.S., Bullmore, E.: Small-world brain networks. The Neuroscientist: a Review Journal Bringing Neurobiology, Neurology and Psychiatry 12(6), 512-523 (2006)

7. Baek, W.H., et al.: Analysis of topological properties in a seismic network. Physica A: Statistical Mechanics and its Applications 391(6), 2279-2285 (2012)

8. Amaral, L., et al.: Classes of small-world networks. Proceedings of the National Academy of Sciences of the United States of America 97(21), 11149-11152 (2000)

9. Vázquez, A., et al.: The topological relationship between the large-scale attributes and local interaction patterns of complex networks. Proceedings of the National Academy of Sciences of the United States of America 101(52), 17940-17945 (2004)

10. Brockmann, D., Helbing, D.: The Hidden Geometry of Complex, Network-Driven Contagion Phenomena. Science 342(6164), 1337-1342 (2013)

11. da Costa, L.F., et al.: Characterization of complex networks: a survey of measurements. Advances in Physics 56(1), 167-242 (2007)

12. Pavlopoulos, G., et al.: Using graph theory to analyze biological networks. BioData Mining 4(1), 10 (2011)

13. Jeong, H., et al.: The large-scale organization of metabolic networks. Nature 407(6804), 651-654 (2000)

14. Ma, H.W., et al.: Hierarchical structure and modules in the Escherichia coli transcriptional regulatory network revealed by a new top-down approach. BMC Bioinformatics 5, 199 (2004) 
15. Milgram, S.: The Small-World Problem. Psychology Today 1(1), 61-67 (1967)

16. Strogatz, S.H.: Exploring complex networks. Nature 410(6825), 268-276 (2001)

17. Newman, M.E., Watts, D.J.: Scaling and percolation in the small-world network model. Physical review. Physical review. E, Statistical Physics, Plasmas, Fluids, and Related Interdisciplinary Topics 60(6 pt. B), 7332-7342 (1999)

18. Zhao, H., et al.: Self-organizing Ising model of artificial financial markets with small-world network topology. EPL (Europhysics Letters) 101(1), 18001 (2013)

19. Moore, C., Newman, M.E.: Epidemics and percolation in small-world networks. Physical review. E, Statistical Physics, Plasmas, Fluids, and Related Interdisciplinary Topics 61(5 pt. B), 5678-5682 (2000)

20. Watts, D.J., Strogatz, S.H.: Collective dynamics of 'small-world' networks. Nature 393(6684), 440-442 (1998)

21. Assenov, Y., et al.: Computing topological parameters of biological networks. Bioinformatics 24(2), 282-284 (2008)

22. Ravasz, E., et al.: Hierarchical organization of modularity in metabolic networks. Science 297(5586), 1551-1555 (2002)

23. Shmulevich, I., Dougherty, E.: From Boolean to probabilistic Boolean networks as models of genetic regulatory networks. Proceedings of the IEEE 90(11), 1778-1792 (2002)

24. Karlebach, G., Shamir, R.: Modelling and analysis of gene regulatory networks. Nature Reviews. Molecular Cell Biology 9(10), 770-780 (2008)

25. Baralla, A., Mentzen, W.I., de la Fuente, A.: Inferring gene networks: dream or nightmare? Annals of the New York Academy of Sciences 1158, 246-256 (2009)

26. Dougherty, E.R., Bittner, M.L.: Causality, randomness, intelligibility, and the epistemology of the cell. Current Genomics 11(4), 221-237 (2010)

27. Lopes, F.M., Ray, S.S., Hashimoto, R.F., Cesar Jr., R.M.: Entropic biological score: a cell cycle investigation for GRNs inference. Gene 541(2), 129-137 (2014)

28. Dougherty, E.R.: Validation of inference procedures for gene regulatory networks. Current Genomics 8(6), 351-359 (2007)

29. Lopes, F.M., Martins Jr., D.C., Barrera, J., Cesar Jr., R.M.: A feature selection technique for inference of graphs from their known topological properties: Revealing scale-free gene regulatory networks. Information Sciences 272, 1-15 (2014)

30. Kauffman, S.A.: Metabolic stability and epigenesis in randomly constructed genetic nets. Journal of Theoretical Biology 22(3), 437-467 (1969)

31. Pudil, P., Novovičová, J., Kittler, J.: Floating search methods in feature-selection. Pattern Recognition Letters 15(11), 1119-1125 (1994)

32. Lopes, F.M., Martins Jr., D.C., Cesar-Jr, R.M.: Feature selection environment for genomic applications. BMC Bioinformatics 9(1), 451 (2008)

33. Lopes, F.M., Martins Jr., D.C., Barrera, J., Cesar Jr., R.M.: SFFS-MR: a floating search strategy for GRNs inference. In: Dijkstra, T.M.H., Tsivtsivadze, E., Marchiori, E., Heskes, T. (eds.) PRIB 2010. LNCS, vol. 6282, pp. 407-418. Springer, Heidelberg (2010)

34. Yu, L., Watterson, S., Marshall, S., Ghazal, P.: Inferring Boolean networks with perturbation from sparse gene expression data: a general model applied to the interferon regulatory network. Molecular BioSystems 4(10), 1024-1030 (2008)

35. Lopes, F.M., Cesar Jr., R.M., da Costa, L.F.: AGN simulation and validation model. In: Bazzan, A.L.C., Craven, M., Martins, N.F. (eds.) BSB 2008. LNCS (LNBI), vol. 5167, pp. 169-173. Springer, Heidelberg (2008)

36. Lopes, F.M., Cesar Jr., R.M., da Costa, L.F.: Gene expression complex networks: Synthesis, identification, and analysis. Journal of Computational Biology 18(10), 1353-1367 (2011) 